\title{
UNIVERSITYOF
}

FORWARD

THINKING

WESTMINSTER用

WestminsterResearch

http://www.westminster.ac.uk/westminsterresearch

\section{Comprehensive HPTLC fingerprinting as a tool for a simplified analysis of purity of ginkgo products}

Frommenwiler, D., Booker, A., Vila, R., Heinrich, M., Reich, E. and Cañiguerala, $\mathbf{S}$.

NOTICE: this is the authors' version of a work that was accepted for publication in Journal of Ethnopharmacology. Changes resulting from the publishing process, such as peer review, editing, corrections, structural formatting, and other quality control mechanisms may not be reflected in this document. Changes may have been made to this work since it was submitted for publication. A definitive version was subsequently published in Journal of Ethnopharmacology, 243, 112084, 2019.

The final definitive version in Journal of Ethnopharmacology is available online at:

https://dx.doi.org/10.1016/j.jep.2019.112084

(C) 2019. This manuscript version is made available under the CC-BY-NC-ND 4.0 license https://creativecommons.org/licenses/by-nc-nd/4.0/

The WestminsterResearch online digital archive at the University of Westminster aims to make the research output of the University available to a wider audience. Copyright and Moral Rights remain with the authors and/or copyright owners.

Whilst further distribution of specific materials from within this archive is forbidden, you may freely distribute the URL of WestminsterResearch: ((http://westminsterresearch.wmin.ac.uk/)).

In case of abuse or copyright appearing without permission e-mail repository@westminster.ac.uk 


\section{Comprehensive HPTLC fingerprinting as a tool for a simplified analysis of purity of ginkgo products}

Débora Arruda Frommenwiler ${ }^{\mathrm{a}, \mathrm{b}}$, Anthony Booker ${ }^{\mathrm{c}, \mathrm{d}}$, Roser Vila ${ }^{\mathrm{a}}$, Michael Heinrich ${ }^{\mathrm{c}}$, Eike Reich and Salvador Cañigueral ${ }^{a^{*}}$

a Unitat de Farmacologia, Farmacognòsia i Terapèutica, Facultat de Farmàcia i Ciències de l'Alimentació, Universitat de Barcelona, Av. Joan XXIII, 27-31, ES-08028 Barcelona - Spain

${ }^{\mathrm{b}}$ CAMAG AG, Sonnenmattstrasse 11, 4132 Muttenz - Switzerland

c Pharmacognosy and Phytotherapy, Biodiversity and Medicines Research Cluster, Department of Pharmaceutical and Biological Chemistry, UCL School of Pharmacy. 29-39 Brunswick Square, WC1N 1AX London - UK

${ }^{d}$ Herbal and East Asian Medicine, Department of Life Sciences, College of Liberal Arts and Sciences, University of Westminster, 115 New Cavendish Street, London, W1W 6UW, UK

*Corresponding author

Salvador Cañigueral, Unitat de Farmacologia, Farmacognòsia i Terapèutica, Facultat de Farmàcia i Ciències de l'Alimentació, Universitat de Barcelona. Av. Joan XXIII, 27-31, ES-08028 Barcelona - Spain Phone: +344024531

Email address: s.canigueral@ub.edu 


\begin{abstract}
Ethnopharmacological relevance: Herbal medicinal products based on ginkgo leaf refined dry extract (GBE) are an European development from the Eastern Asia traditionally used species Ginkgo biloba L. Nowadays, ginkgo products have increased the presence in the market, mainly as dietary supplements. Its adulteration with rutin and quercetin or herbal extracts rich in these compounds is a common practice. Tests featuring assays and detection of adulterants need to be performed on top of other existent methods (e.g. identification test). This may increase the costs of evaluating the quality of ginkgo products.
\end{abstract}

Aim of the study: To prove that comprehensive HPTLC fingerprinting can provide information beyond identification of ginkgo products, avoiding additional chromatographic tests for detection of adulterations.

Materials and methods: The information contained in the fingerprint obtained by HPTLC analysis of flavonoids was used for identification and for detection of adulterants, as well as to verify the limits of rutin and quercetin, which are normally determined by HPLC and used for detection of adulterants. For this purpose, peak profiles were generated from HPTLC chromatogram images. USP-HPLC methods were used for quantification of total flavonoids and testing the limits of rutin and quercetin. HPLC data were used to support the validity of the HPTLC method. An additional reversed phase HPTLC method was developed as a possible confirmatory method for the quercetin limit test.

Results: The proposed HPTLC method uses a particular sequence of detections, resulting in a number of images, which are later interpreted in a certain order. It is able to identify ginkgo products, to detect adulterants (rutin, quercetin, sophora fruit and flower bud, and buckwheat), and, using peak profiles generated from the chromatogram images prior to and after derivatisation, to evaluate the limits of rutin and quercetin. Forty-eight out of fifty-nine ginkgo dietary supplements analysed contained one or more adulterants. Furthermore, results of the HPTLC and HPLC limit tests for rutin and quercetin were in agreement in $98 \%$ of the cases. Finally, a decision tree showing the sequence of interpretation of the fingerprints obtained with the different detections after a single HPTLC analysis is included to help the analyst to evaluate whether samples have the correct identity and whether they contain or not adulterants. 
Conclusion: A single HPTLC analysis is able to provide information on identity and purity of the products. This simplifies the analytical workflow and reduces the number of analyses prescribed in the USP powdered ginkgo extract monograph.

Keywords: HPTLC; Ginkgo biloba L.; comprehensive HPTLC fingerprinting; identity; adulteration, dietary supplements;

\section{ABBREVIATIONS}

BWH: Buckwheat herb

GBE: Ginkgo leaf refined dry extract

GBL: Ginkgo leaf

GP: Ginkgo product

HPTLC: High-Performance Thin-Layer Chromatography

I: Isorhamnetin

K: Kaempferol

NLT: Not less than

NP: Natural products reagent

PEG: Polyethylene glycol reagent

Q: Quercetin

SST: System suitability test

SJfr: Sophora fruit

SJfl: Sophora flower bud

USP: United States Pharmacopeia 


\section{Introduction}

Ginkgo (Ginkgo biloba L.), considered a sacred tree in the Eastern Asia, is traditionally associated with longevity. The earliest known medicinal use of ginkgo dates back to $2800 \mathrm{BC}$ and it is described for the pseudofruits, which are more frequently used than leaves in Eastern Asia. However, based on some not very well documented uses in traditional Chinese medicine (TCM), a German company developed a medicinal product from leaves for cognitive impairment in dementia. The active ingredient is a 'special' extract - extract G. biloba (EGb) 761 -obtained by a water-acetone extraction and subsequent purification (Heinrich, 2010, Drieu and Jaggy, 2000). This ginkgo leaf refined dry extract (GBE), which uses $50 \mathrm{~kg}$ of leaf to yield $1 \mathrm{~kg}$ of extract, is standardized to contain 22-27\% of flavonol glycosides and 5.4-6.6\% of terpene lactones (EDQM, 2018), and it is accepted for the improvement of age-associated cognitive impairment and of quality of life in mild dementia (European Medicines Agency, 2015). EGB products (with EGb 761 and other similar refined extracts), that nowadays are in the market either as medicinal products or as dietary supplements, are in high demand. The global trade of gingko leaf extracts has registered a consistent growth in the last years. However, to produce such extract, about 19 steps are involved with costs estimated at \$200-250/Kg (Gafner, 2018, Czigle et al., 2018).

Because of the complexity and high cost of the manufacturing process, adulteration of ginkgo products (GP) has become an important problem in poorly regulated markets. Booker et al. (2016) evaluated the quality of GP sold as food supplements in the UK, revealing that the majority of the samples (32 out of 35) showed a HPTLC fingerprint different from that described in the European Pharmacopoeia and US Pharmacopoeia. It was hypothesized they were adulterated with rutin, quercetin, and other botanical materials. One sample was found to contain only 5hydroxytryptophan.

According to Gafner (2018), industry experts agree that adulteration of GP often stems from economic considerations. Several publications report the addition of flavonol aglycones quercetin and/or kaempferol and/or the flavonol glycoside rutin to ginkgo products. Such practice is performed to ensure that those products, containing less extract, will have higher levels of total flavonoids after hydrolysis (22-27\%) and thus comply with the HPLC assay specifications of the Pharmacopoeias (The United States Pharmacopoeia (USP), 2016b). The assay is based on the hydrolysis of the glycosides and the quantification of the resulting aglycones. Consequently, other botanical material, rich in glycosides of the same flavonol aglycones have also been reported as 
adulterants. They are sophora fruit and flower bud (Styphnolobium japonicum (L.) Schott, syn: Sophora japonicum L.) and buckwheat herb (Fagopyrum sp Moench) (Avula et al., 2015; Chandra et al., 2011; Franz et al., 2011; He and Roller, 2011; Lopez-Gutierrez et al., 2016; Ma et al., 2016; Tawab et al., 2010; Wohlmuth et al., 2014; Yang et al., 2016, Gafner, 2018).

Due to the lack of specificity to detect adulterants of the flavonoid-based assays, several publications propose additional methods, using different chromatographic techniques and detections (López-Gutiérrez et al., 2016, van Beek and Montoro, 2009). Since 2015, the United States Pharmacopoeia (USP) introduced an additional HPLC limit test for rutin and quercetin to the ginkgo extract monograph (The United States Pharmacopoeia (USP), 2016b). As acceptance criteria, the test solution (prior to hydrolysis) should not contain more than $4 \%$ of rutin and $0.5 \%$ of quercetin. As both substances have very different polarities, a gradient elution system over 1 hour is needed. To perform all tests for identity, purity, and strength according to USP, five different analyses (including HPTLC and HPLC) featuring five different sample preparations, to analyse three classes of compounds are required. However, if identification is performed by HPTLC based on the "comprehensive HPTLC fingerprinting" concept (Frommenwiler et al., 2018), more information can be obtained in a single analysis, and so avoid one HPLC test for purity.

In this paper we aim at further developing and illustrating how HPTLC fingerprints can be generated primarily for identification, in order to deliver additional information regarding the

presence of adulterants and specifically the content of rutin and quercetin in ginkgo products. For detection of adulterants, different derivatisation steps and detection modes are considered. Additionally, quantitative information of the profiles generated from the images are used to verify compliance with the limit test for rutin and quercetin. The generated data are compared to those obtained by HPLC. A decision-tree detailing how best to perform comprehensive HPTLC fingerprinting for identification and detection of adulterations in gingko products is proposed.

\section{Experimental}

\subsection{Samples and reference substances}

Fifty-nine products of Ginkgo, including tablets, capsules, and soft gel capsules (GP1-PG59), sold as food supplements or medicines were used in the present work. They declared to contain either refined extracts (22-27\% of flavonol glycosides), extracts with either DER 50:1 or no additional 
information, powdered leaf, extract and leaf, extract and rutin, or extract and green buckwheat. A detailed description of the samples is presented in the supplementary information, Table S1.

Ginkgo leaf reference extracts (GBE) were obtained from Sigma/HWI (Darmstadt, Germany) and Dr. Willmar Schwabe (Karlsruhe, Germany). A sample of ginkgo leaf (GBL) was provided by the American Herbal Products Association (AHPA, Silver Spring, MD, USA). Botanical reference material of sophora fruit and flower bud were obtained from American Herbal Pharmacopoeia (AHP, Scotts Valley, USA). Buckwheat herb (batch 155239) was obtained from Chrüterhüsli (Basel, Switzerland). The standards genistein (99\%), quercetin $(99.5 \%)$ and rutin $(91.3 \%)$ were obtained from USP (Rockville, USA). Kaempferol (97\%) was obtained from Sigma (Darmstadt, Germany) and USP. Isorhamnetin (99\%) from Extrasynthese (Genay, France) and USP.

\subsection{Reagents and solvents}

Polyethylene glycol 400 (99.5\%), 2-aminoethyldiphenylborinate (96.5\%), acetic acid (99.5\%), toluene ( $\geq 99 \%$ ), acetone (pure), dichloromethane (HPLC grade), sodium acetate anhydrous (99.5\%) and acetic anhydride (98.5\%) were purchased from Merck (Darmstadt, Germany). Methanol (HPLC grade) and ortho-phosphoric acid (85\%) were purchased from Carl Roth GmbH (Karlsruhe, Germany). Acetonitrile (HPLC grade), ethyl acetate (99.5\%), formic acid ( $\geq 98 \%$ ), tetrahydrofuran (extra pure) and $p$-anisaldehyde ( $\geq 99 \%)$ were purchased from Acros (New Jersey, USA). Ethanol (99.9\%) was purchased from Alcosuisse (Bern, Switzerland). Deionized water was generated in-house. For the HPLC analysis, methanol, acetonitrile (both HPLC gradient grade) and hydrochloric acid (37\%) were purchased from Fisher Scientific (Hamptom, USA). Phosphoric acid $(\geq 85 \%)$ was obtained from Honeywell (New Jersey, USA), and formic acid (98-100\%) from Scharlau (Barcelona, Spain).

\subsection{Instruments}

A CAMAG HPTLC system controlled by visionCATS software and including Visualizer, Automatic TLC Sampler 4, Automatic Developing Chamber 2, TLC Plate Heater III and Chromatogram Immersion device was used.

For the HPLC analysis, a Shimadzu instrument of the UFLC series c including auto sampler SIL20AC HT, pumps LC-20AD, degasser DGU-20As, column oven CTO-20A, diode array detector

SPD-M20A, Phenomenex Gemini® NX C18 (100 ̊̊, $5 \mu \mathrm{m}, 4.6 \mathrm{~mm} \times 250 \mathrm{~mm})$ column and communication bus module CBM-20A was used. The software was LC Solutions (Shimadzu Corporation) version $1.23 \mathrm{SP} 1$. 


\subsection{High performance thin layer chromatography (HPTLC)}

HPTLC parameters for plate layout, sample application, conditioning of the plate, plate development and visualization were in agreement with the USP general Chapter <203> (The United States Pharmacopoeia (USP), 2017). The quality of the chromatography was verified based on the position of two or three standards, used as system suitability test (SST). The image's background normalization was adjusted over the SST.

\subsubsection{Standard solutions}

Solutions of rutin, chlorogenic acid, and quercetin were prepared at concentration of $0.2 \mathrm{mg} / \mathrm{mL}$ in methanol for the SST. For the limit test of rutin, the standard solution was prepared at $0.4 \mathrm{mg} / \mathrm{mL}$ of rutin in methanol. For the quercetin limit test based on a reference extract (Sigma/HWI GBE was used in this case), the weight of extract was adjusted to yield a solution (in methanol) representing $0.5 \%$ of quercetin in the extract. For the limit test of quercetin on reversed phase, the standard solution was prepared at $0.05 \mathrm{mg} / \mathrm{mL}$ of quercetin in methanol.

\subsubsection{Test solutions}

Sample of gingko leaf and products containing leaf were prepared according to the USP Ginkgo monograph (The United States Pharmacopoeia (USP), 2016a). Products containing ginkgo extract (capsules, soft gel capsules and tablets) were prepared to contain $10 \mathrm{mg}$ of extract per $\mathrm{mL}$ of methanol, sonicated for 10 minutes at room temperature and centrifuged for 5 minutes at 5000 rpm. Products containing leaf plus extract were processed as leaf, taking in account the labelled content of extract and the drug-extract ratio, which were used to calculate its equivalence as leaf.

\subsubsection{Chromatography}

The HPTLC method used for evaluating the samples was based on the USP ginkgo and powdered ginkgo extract monographs (The United States Pharmacopoeia (USP), 2016a and 2016b). The parameters for application volume, detection and derivatisation methods were optimized (see parameters described under HPTLC ID method in Table 1). A confirmatory additional method for the limit of quercetin was developed using a reversed phase plate (parameters are also described in Table 1).

Table 1.

Parameters for the two HPTLC methods used in the present work. 


\begin{tabular}{|c|c|c|}
\hline Parameters & ID method & Limit of quercetin (reversed phase) \\
\hline Stationary phase & 20x10cm plates Si $60 \mathrm{~F}_{254}$ (Merck) & 20x10cm plates Si $60 \mathrm{RP}-18 \mathrm{~W}$ (Merck) \\
\hline SST & $0.2 \mathrm{mg} / \mathrm{mL}$ of rutin, quercetin and chlorogenic acid & $0.2 \mathrm{mg} / \mathrm{mL}$ of rutin and quercetin \\
\hline $\begin{array}{l}\text { Preparation of } \\
\text { standards for limit test }\end{array}$ & $0.4 \mathrm{mg} / \mathrm{mL}$ (rutin, limit test) & $0.05 \mathrm{mg} / \mathrm{mL}$ (quercetin, limit test) \\
\hline Application volume & $3 \mu \mathrm{L}$ of test and standards solutions & $3 \mu \mathrm{L}$ of test and standards solutions \\
\hline Developing solvent & $\begin{array}{l}\text { Ethyl acetate, acetic acid, formic acid, water } \\
\qquad(100: 11: 11: 26 \mathrm{~V} / \mathrm{V} / \mathrm{V} / \mathrm{V})\end{array}$ & $\begin{array}{c}\mathrm{ACN}^{\mathrm{a}}, \mathrm{THF}^{\mathrm{b}} \text { and } 0.5 \% \mathrm{H}_{3} \mathrm{PO}_{4} \text { aqueous solution } \\
(10: 40: 50 \mathrm{~V} / \mathrm{V} / \mathrm{V})\end{array}$ \\
\hline Development & $\begin{array}{l}20 \text { min saturation, } 10 \text { min conditioning at } 33 \% \\
\text { relative humidity (with } \mathrm{MgCl}_{2} \text { ), } 70 \mathrm{~mm} \text { distance } \\
\text { from lower edge, room temperature }=23-27 \square \mathrm{C}\end{array}$ & $\begin{array}{l}20 \text { min saturation, } 10 \mathrm{~min} \text { conditioning at } 33 \% \\
\text { relative humidity (with } \mathrm{MgCl}_{2} \text { ), } 70 \mathrm{~mm} \text { distance } \\
\text { from lower edge, room temperature }=23-27 \square \mathrm{C}\end{array}$ \\
\hline $\begin{array}{l}\text { Documentation prior } \\
\text { to derivatisation }\end{array}$ & UV $254 \mathrm{~nm}, \mathrm{UV} 366 \mathrm{~nm}$ & Not applicable \\
\hline Derivatisation 1 & $\begin{array}{c}\text { Plates were heated at } 100 \square \mathrm{C} \text { for } 3 \mathrm{~min} \text { and } \\
\text { derivatised by dipping (speed: } 3 \text {, time: } 0 \text { ) in NP } \\
\text { reagent and then in PEG reagent }\end{array}$ & $\begin{array}{c}\text { Plates were heated at } 100 \square \mathrm{C} \text { for } 3 \mathrm{~min} \text { and } \\
\text { derivatised by dipping (speed: } 3 \text {, time: } 0 \text { ) in } \mathrm{NP} \\
\text { reagent and then in PEG reagent }\end{array}$ \\
\hline
\end{tabular}

\begin{tabular}{cc}
\hline Derivatisation 2 & Plates were heated at $100 \square \mathrm{C}$ for 3 min and \\
derivatised by dipping (speed: 3 , time: 0 ) in NP & Not applicable \\
reagent
\end{tabular}

\section{Documentation after}

derivatisation 1 or 2

UV $366 \mathrm{~nm} \quad$ UV $366 \mathrm{~nm}$

\begin{tabular}{lcc}
\hline Derivatisation 3 & $\begin{array}{c}\text { Plates were dipped (speed: 3, time: 0) in p- } \\
\text { anisaldehyde reagent and heated at } 100 \square \mathrm{C} \text { for 3 } \\
\text { min }\end{array}$ & Not applicable \\
\hline $\begin{array}{l}\text { Documentation after } \\
\text { derivatisation 3 }\end{array}$ & White light & Not applicable \\
\hline
\end{tabular}

${ }^{\mathrm{a} A C N}$ : acetonitrile; ${ }^{\mathrm{b}} \mathrm{THF}$ : tetrahydrofuran;

\subsubsection{Derivatisation reagents}

Natural products reagent (NP) was prepared dissolving $1 \mathrm{~g}$ of 2-aminoethyl diphenylborinate in $200 \mathrm{~mL}$ of ethyl acetate. As a polyethylene glycol reagent (PEG) a dissolution of $10 \mathrm{~g}$ of polyethylene glycol 400 in $200 \mathrm{~mL}$ of dichloromethane was used. Finally, the anisaldehyde reagent was obtained dissolving $1 \mathrm{~mL}$ of p-anisaldehyde in $200 \mathrm{~mL}$ of a mixture of methanol, acetic acid and sulphuric acid (170:20:10 V/V/V).

\subsubsection{Generation of peak profiles from chromatogram images}


Where necessary, the visionCATS software was used to generate peak profiles from fingerprints by calculating the luminance as $L=(1 / 3 \mathrm{R})+(1 / 3 \mathrm{G})+(1 / 3 \mathrm{~B})$ from the average of red $(\mathrm{R})$, green $(\mathrm{G})$, and blue (B) pixels of each line of the track and then plotting it against the $R_{\mathrm{F}}$ values (Frommenwiler et al., 2018).

\subsubsection{Limit tests of rutin and quercetin}

Peak profiles were used for performing the limit tests of rutin and quercetin. Detections under UV $254 \mathrm{~nm}$ prior to derivatisation (rutin) and under UV $366 \mathrm{~nm}$ after derivatisation with NP or NP plus PEG (quercetin) were used. Calculations were based on the peak heights at the position of rutin or quercetin in the samples and the standard solutions. Standard solutions at concentrations corresponding to the maximum limit accepted for rutin and quercetin according to USP powdered ginkgo extract monograph were used.

\subsubsection{Validation of the limit test of rutin and quercetin using the HPTLC ID method}

The analytical procedure, presented in this research has been validated in order to confirm its reliability. Linearity was established for rutin, based on the peak area of the peak profile under UV $254 \mathrm{~nm}$ prior to derivatisation. Six data points in the range of $0.75-1.8 \mu \mathrm{g} /$ application with 0.15 and $0.3 \mu \mathrm{g}$ intervals were used. The correlation coefficient $\left(\mathrm{R}^{2}\right)$ was 0.999050 and the coefficient of variation $(\mathrm{CV})$ was $0.88 \%$. For quercetin, an extract was used to build the calibration curve. The peak heights of the peak profile under UV $366 \mathrm{~nm}$ after derivatisation were used. Linearity of the zone at the position of quercetin was established using six data points in the range of 0.06$0.21 \mu \mathrm{g} /$ application of quercetin with $0.03 \mu \mathrm{g}$ intervals, using GB reference extract with known content of quercetin $(0.297 \%)$. The correlation coefficient $\left(\mathrm{R}^{2}\right)$ was 0.999223 and the coefficient of variation (CV) was $1.51 \%$. For intraday and interday precision, one GB reference extract was prepared 4 times (each day), and solutions were analysed twice ( 2 plates per day). The absolute area of the peak at the position of rutin (under UV $254 \mathrm{~nm}$ prior after derivatization) and absolute height of the peak at the position of quercetin (under UV $366 \mathrm{~nm}$ prior to derivatization) in GB extract was used for the precision test. The intraday CV\% was 2.08 for rutin and 0.89 for quercetin. The interday CV\% was 3.48 for rutin and 2.65 for quercetin.

\subsection{High performance liquid chromatography (HPLC)}


The HPLC assay of total flavonoids was performed according to the USP powdered ginkgo extract monograph (The United States Pharmacopoeia (USP), 2016b) with all samples that declare to contain GBE. Samples that contain leaf were analysed according to its specific monograph. For tablets and capsules, an initial weight equivalent to $300 \mathrm{mg}$ of extract was used. Additionally, the specific tests (limit of rutin and quercetin) according to USP powdered ginkgo extract monograph were applied for products containing refined extracts or other extracts. For products containing ginkgo leaf or mixtures of extracts with ginkgo leaf, rutin or buckwheat, this limit test was not performed. For tablets and capsules, an initial weight equivalent to $100 \mathrm{mg}$ of extract was used. For both, assay and limit tests, each sample was prepared and analysed in duplicate.

\section{Results and discussion}

In the current study, thirty-five ginkgo products obtained in the UK and twenty-four other products from other countries were analysed particularly for their purity. In addition to the detection prescribed in the identification section of the monographs, other detections were used (UV $254 \mathrm{~nm}$ and $366 \mathrm{~nm}$ prior to derivatisation). Those multiple images, generated during the identification test, are available for each analysis. HPTLC fingerprints and results for identity and adulteration of all samples are presented in the supplementary information (Figs. S1-S4, Table S2). In a first set of experiments, fifty-nine samples of ginkgo products were evaluated with the HPTLC ID method. Their fingerprints were compared to the description of the monograph and to reference materials of ginkgo leaf and extract. Typical fingerprints are shown in Fig. 1. It was observed that only eleven samples were compliant with the monograph description.

Under UV $254 \mathrm{~nm}$ prior to derivatisation (Fig. 1), several products show intense quenching zones at the position of rutin (e.g. GP17), or at $R_{\mathrm{F}} \mathrm{s} 0.12$ and/or 0.6 (e.g. GP4). The two latter zones are absent in ginkgo. Under UV $366 \mathrm{~nm}$ prior to derivatisation (Fig. 1), red zones due to chlorophylls are seen in GBL. Additionally, GBL and GBE show a blue fluorescent zone at the position of chlorogenic acid, characteristic of ginkgo. Four products lacked this zone (e.g. GP4 and GP7) and other four products showed it, but fainter than GBL and GBE. Under UV $366 \mathrm{~nm}$ after derivatisation 1 (Fig. 1), twenty products showed an intense yellow zone at the position of quercetin (e.g. GP4 and GP5) and sometimes faint zones in the rest of the fingerprint (e.g. GP17). 


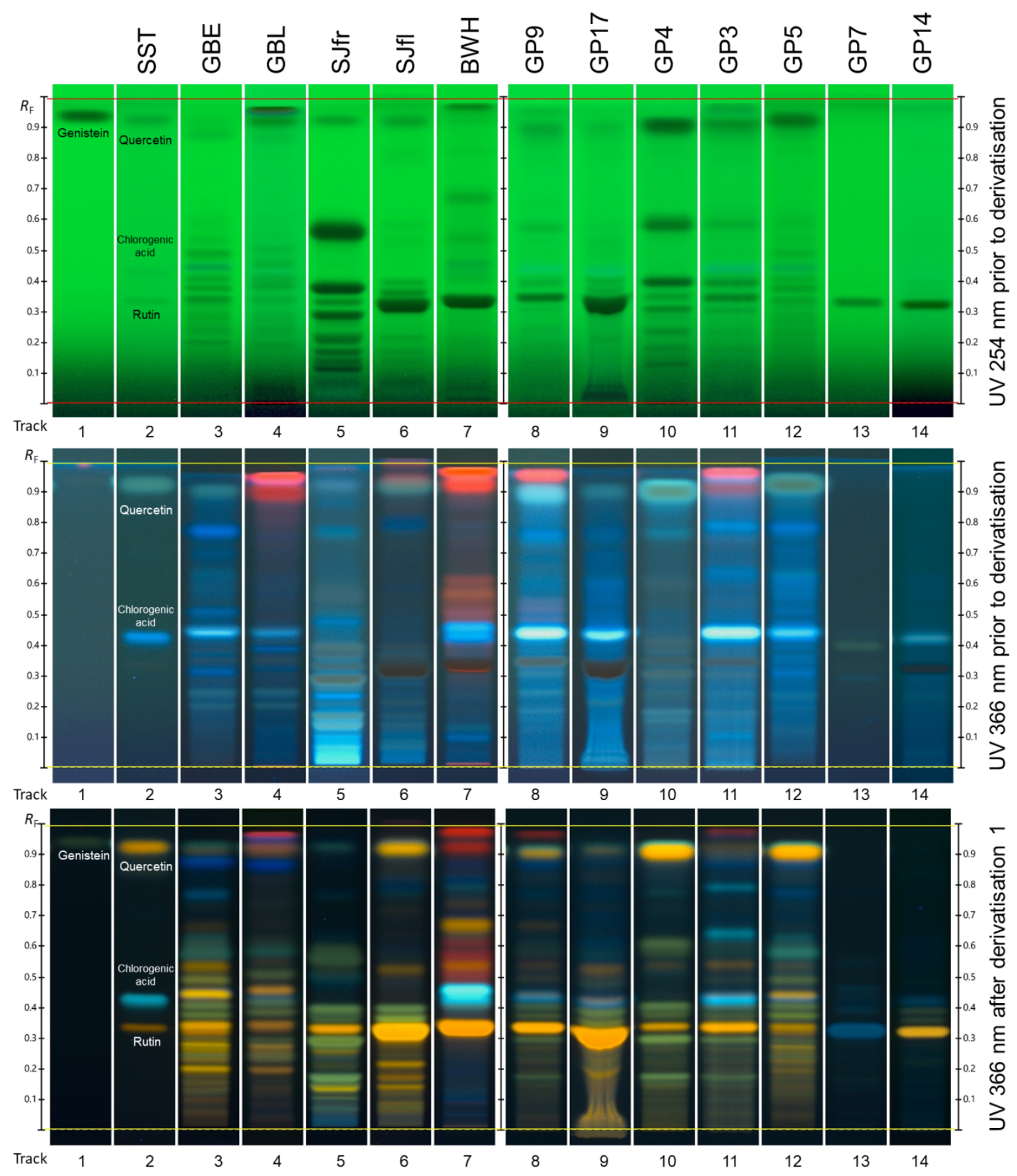

Fig. 1. HPTLC fingerprints of reference standards, ginkgo leaf and extract, sophora fruit, sophora flower buds, buckwheat herb and different cases of adulterations of ginkgo products under different detection modes. Track 1: genistein; SST: system suitability test (rutin, chlorogenic acid, and quercetin with increasing $R_{\mathrm{F}}$ values); GBE: ginkgo leaf refined dry extract; GBL: ginkgo leaf; SJfr: Sophora fruit; SJfl: Sophora flower bud; BWH: Buckwheat herb; GP: ginkgo product. 
Eight products lacked zones characteristic of ginkgo and showed mainly a zone due to rutin (e.g. GP14). One sample contained only 5-hydroxy tryptophan (GP7) as shown previously. One sample (GP3) showed an additional blue zone between $R_{\mathrm{F}} \mathrm{S} 0.6$ and 0.7. Sixteen samples showed additional green zones between $R_{\mathrm{F}} \mathrm{S} 0.2$ and 0.3 (e.g. GP17, GP4 and GP3) most of the time combined with high levels of quercetin. Of the five samples labelled as containing ginkgo leaf, two yielded fingerprints similar to that of GBE, while three corresponded to GBL, but the zones were less pronounced.

Based on the identification results, it was concluded that many of the analysed products seemed to have quality problems, including adulteration. In addition, the intensity of some fingerprints was lower than expected. To confirm the hypothesis that some of the products had less extract than declared, their total flavonoids content was evaluated.

\subsection{HPLC analysis of flavonoids in ginkgo products according to the USP}

The content of flavonol glycosides was determined in fifty-three ginkgo products (GP). Five products were not analysed by HPLC due to limited amount of sample available. The percentage of flavonoids was calculated from the content of the corresponding aglycones obtained after hydrolysis, which are quercetin (Q), kaempferol (K), and isorhamnetin (I). Acceptable contents of flavonoids, expressed as flavonol glycosides, were $22.0 \%-27.0 \%$ for the extract, and not less than $0.5 \%$ for the leaves. Furthermore, the peak area ratios of K/Q (required $\geq 0.7$ ) and I/Q (required $\geq$ 0.1) were calculated for the products containing extract, as described in the USP powdered ginkgo extract monograph. Forty-four samples that declared to contain extract were also analysed for their content of rutin and quercetin, based on the method specified in the USP. Acceptable contents prior to hydrolysis were $\leq 4 \%$ for rutin and $\leq 0.5 \%$ for quercetin (see Table S3; supplementary information).

The investigated products fall into five categories according to their claims: refined extracts with declaration of total flavonoid content, refined extracts without declaration, mixture of extract and additional ingredient, other extracts, and products based on dried leaves.

Of the thirty-eight products containing refined extract with a declaration of flavonoid content, thirty-four were analysed for their flavonol glycosides content. Of those, twenty-seven contained the amount of flavonol glycosides declared on their labels and twenty-one complied with the K/Q and I/Q peak ratios. Six products had less than $22 \%$ of total flavonoids and one had more than 
$27 \%$. Regarding the rutin and quercetin contents of those thirty-four products, four had more than $4 \%$ of rutin and twenty-two had more than $0.5 \%$ of quercetin. Thus, GP20, 36-43 and 59 were the only products of this group compliant with the USP specifications concerning HPTLC fingerprint, flavonol glycosides content by HPLC, K/Q and I/Q ratios and HPLC limit test of rutin and quercetin.

Eight of the nine products containing refined extract without declaring the flavonoid content were analysed. All of them had less than $22.0 \%$ of flavonol glycosides by HPLC. Of those, two had more than $4 \%$ of rutin and five had more than $0.5 \%$ of quercetin.

Of the five products that declared to contain extract mixed with other ingredients, four were analysed. They declared to contain either rutin, buckwheat, or ginkgo leaf. The products that contained additional rutin or buckwheat had more than $27.0 \%$ of flavonol glycosides, yet are compliant with their label claims.

Of the two products containing other type of extracts, both had less than $22.0 \%$ of flavonol glycosides as determined by HPLC. Finally, all five products declaring to contain ginkgo leaf complied with the total flavonoid content for the leaf (NLT than $0.5 \%$ ).

In general, most of the products that were compliant with the total flavonoids assay had weak fingerprints by HPTLC and/or intense zones at the positions of quercetin or rutin and/or additional zones. To properly detect addition of rutin and/or quercetin, a supplementary HPLC test is required. Nevertheless, results of the HPLTC ID method pointed to quality issues in the same samples as the combined HPLC tests.

In this context, our goal was to evaluate, whether the HPTLC fingerprint, obtained with the ID method, can also be used as tool to determine the purity of ginkgo products by detecting the presence of adulterants.

\subsection{HPTLC analysis of ginkgo products}

In the next steps of the investigation, first a literature review was performed to identify possible adulterants. Then, their HPTLC fingerprints were compared with those of GBL and GBE. Thereafter, physical mixtures of GBL and adulterants were analysed. It was evaluated whether the HPTLC ID method is capable of distinguishing the adulterants from ginkgo and what their respective detection limits were in mixtures. Finally, the HPTLC ID method was evaluated for 
suitability to verify compliance with the USP HPLC limit tests for rutin and quercetin. In that step, different detection modes and derivatisation reagents were investigated.

Samples of sophora fruit and flower bud, and buckwheat were individually analysed using the HPTLC ID method and their fingerprints were compared to those of GBE extract and GBL (Fig. $1)$.

Under UV $366 \mathrm{~nm}$ after derivatisation, sophora fruit featured several unique greenish zones in the lower half of the chromatogram, a yellow zone at the position of rutin and a faint greenish zone at the position of quercetin, kaempferol, and isorhamnetin. The fingerprint of sophora flower bud was comparatively rich in rutin and quercetin, and contained other flavonoids and phenolic compounds (faint zones). Its fingerprint pattern was somewhat similar to that of the GBE. Buckwheat also showed an intense zone at the position of rutin and another blue fluorescent zone between $R_{\mathrm{F}} \mathrm{S} 0.4$ and 0.5. Other reddish and yellowish zones were observed in the upper half of the chromatogram. Other individual characteristics were observed under UV $254 \mathrm{~nm}$ for each sample.

For the investigation of admixtures, physical mixtures of 1, 3, 5, 10, 15, and 20\% each of sophora fruit, sophora flower bud, and buckwheat powdered drugs with 99, 97, 95, 90, 85, and 80\% of ginkgo leaf were prepared.

\subsubsection{Admixture of buckwheat herb (Fagopyrum sp.)}

To visualise admixtures with buckwheat, different detection modes were evaluated. Under UV 366 $\mathrm{nm}$ prior to derivatisation (Fig. S5), a faint reddish zone was seen in the middle of the chromatogram in the ginkgo leaf spiked with $15 \%$ buckwheat. This zone was absent in ginkgo leaf. One sample (GP9), which listed buckwheat as an ingredient, showed similar reddish zones. In this respect, the product cannot be considered as adulterated. Nevertheless, the same sample was found to contain sophora flower bud.

\subsubsection{Admixture of sophora flower bud}

When sophora flower bud was mixed with ginkgo leaf (Fig. 2), the amounts of rutin and quercetin increased. However, with this method it was not possible to link these higher levels of rutin and quercetin to bulk chemicals or a herbal drug. In the absence of specific markers to detect the presence of sophora flower bud, alternative derivatisation 3 was introduced: following derivatisation with NP reagent and visualising the image under UV $366 \mathrm{~nm}$, the plate was derivatised with anisaldehyde reagent and documented under white light. Because anisaldehyde 
reagent cannot be used subsequently to PEG reagent (used in derivatisation 1 of the ID method), that derivatisation reagent had to be eliminated (derivatisation 2) (Table 1). Detection of flavonoids is not significantly altered by PEG (Fig. S6). However, subsequent use of anisaldehyde instead provided an extra set of data without the need to repeat the chromatography.

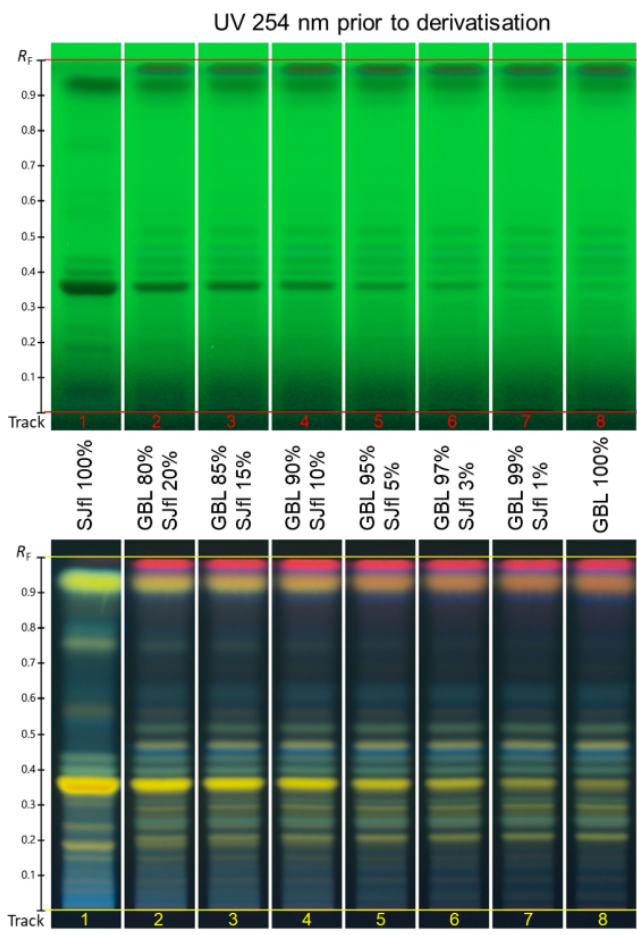

UV $366 \mathrm{~nm}$ after derivatisation 2
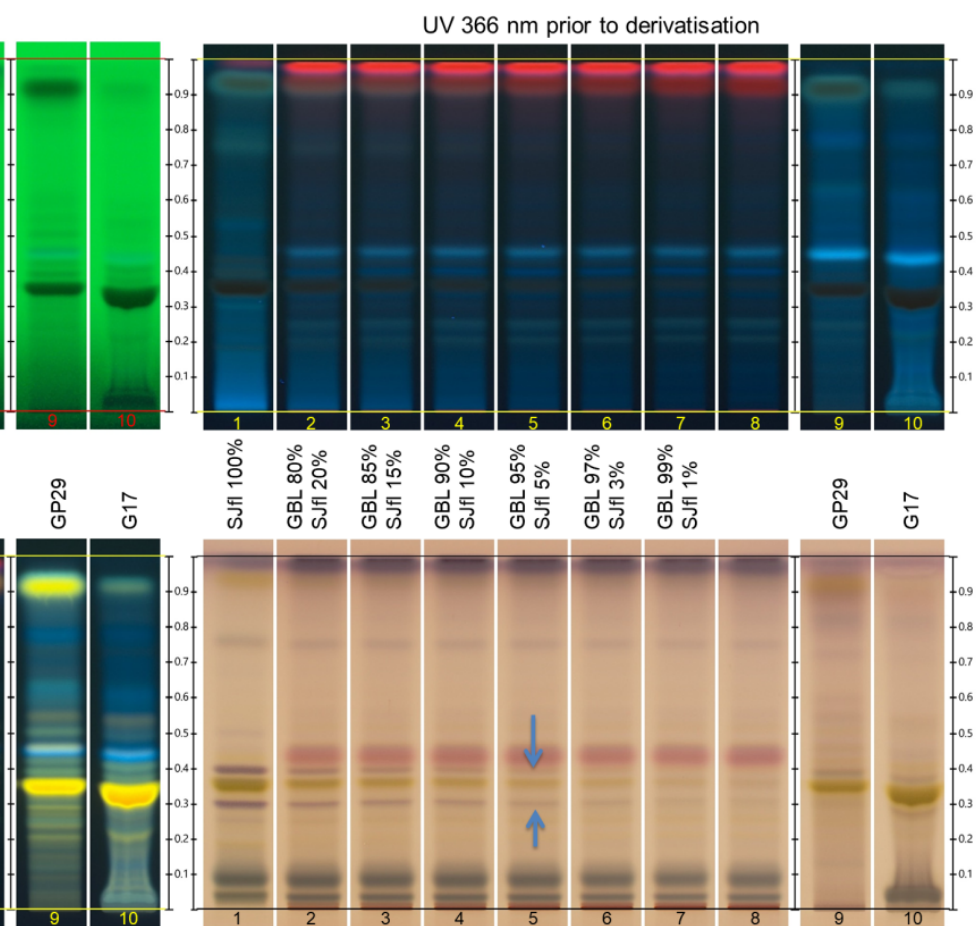

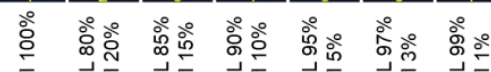
要

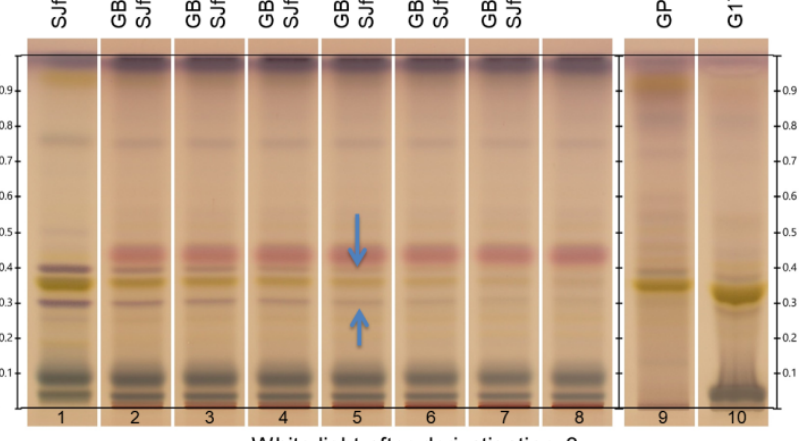

White light after derivatisation 3

Fig. 2. HPLTC fingerprints of physical mixtures of ginkgo leaf and sophora flower bud, and examples of adulterated products. GBL: ginkgo leaf, SJfl: sophora flower bud. Track 1: 100\% SJfl, track 8: 100\% GBL, tracks 2-8: mixtures of SJfl and GBL, tracks 9-10: products adulterated with SJfl.

The chromatogram of ginkgo leaf spiked with $5 \%$ of sophora flower bud showed two purple zones above and below the zone due to rutin, combined with an elevated amount of rutin. When the ginkgo products were tested with this detection, two samples (GP29 and 17) presented a pattern similar to that described above, indicating adulteration with sophora flower bud.

\subsubsection{Admixture of sophora fruit}

Avula et al. (2015) and Wohlmuth et al. (2014) stated that genistein is a characteristic constituent of sophora fruit and can be used as a marker to detect adulteration with this drug. However, in the HPTLC ID method genistein migrated close to the solvent front and was barely detected after 
derivatisation under UV $366 \mathrm{~nm}$ (Fig. 1). Under UV $254 \mathrm{~nm}$ prior to derivatisation, genistein was detectable (Fig. 1) but co-eluted with quercetin.

In Fig. 3, the fingerprint of ginkgo leaf containing 1\% of sophora fruit after derivatisation, showed a green zone between $R_{\mathrm{F}} \mathrm{S} 0.1$ and 0.2 , which was absent in $100 \%$ ginkgo leaf. Under UV $254 \mathrm{~nm}$ prior to derivatisation one quenching zone between $R_{\mathrm{F}} \mathrm{S} 0.5$ and 0.6 was seen in the sample spiked at $1 \%$. A second zone between $R_{\mathrm{F}} \mathrm{S} 0.1$ and 0.2 was observed in the sample spiked at $3 \%$. The described zones are detected in 17 of the 59 products. Therefore, these three zones can be used to identify the presence of $1 \%$ or more of sophora fruit in mixtures with ginkgo leaf.

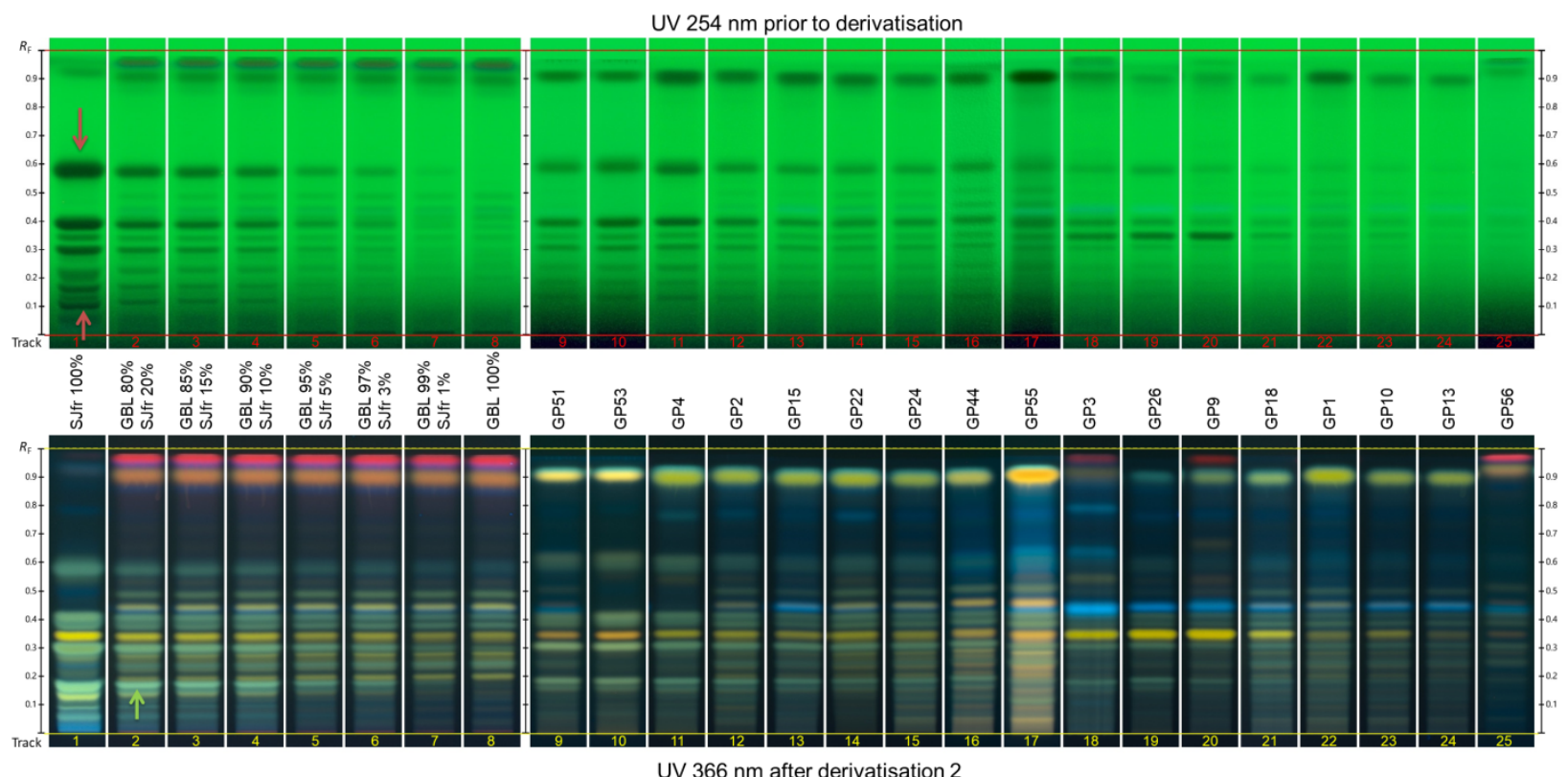

Fig. 3. HPLTC fingerprints of the physical mixtures of ginkgo leaf and sophora fruit, and examples of adulterated products. Track 1: 100\% SJfr, track 8: 100\% GBL, tracks 2-8: mixtures of SJfr and GBL, tracks 9-25: products adulterated with SJfr.

\subsubsection{Limit test for rutin}

Forty-six products declaring to contain ginkgo extract, were investigated for compliance with the USP limit for rutin by the HPTLC ID method using a reference solution containing rutin standard at $0.4 \mathrm{mg} / \mathrm{mL}$, equivalent to $4 \%$ of the extract weight. Percentage values of rutin were calculated. Results are shown in Fig. 4 and Table S2. Eight samples (GP14, 17, 23, 26, 32, 45, 49 and 54) exceeded the 4\% limit. Of those, samples GP14, 23, 32, 4549 and 54 seemed to contain mainly rutin, because they showed only a yellow zone at the position of rutin and lacked other zones 
characteristic of ginkgo. Samples GP17 and 26 were found to be adulterated with sophora flower bud and fruit, and consequently showed an amount of rutin above the accepted level. One sample that contained mainly rutin (GP 30) complied with the limit test and had a low content of flavonol glycosides.

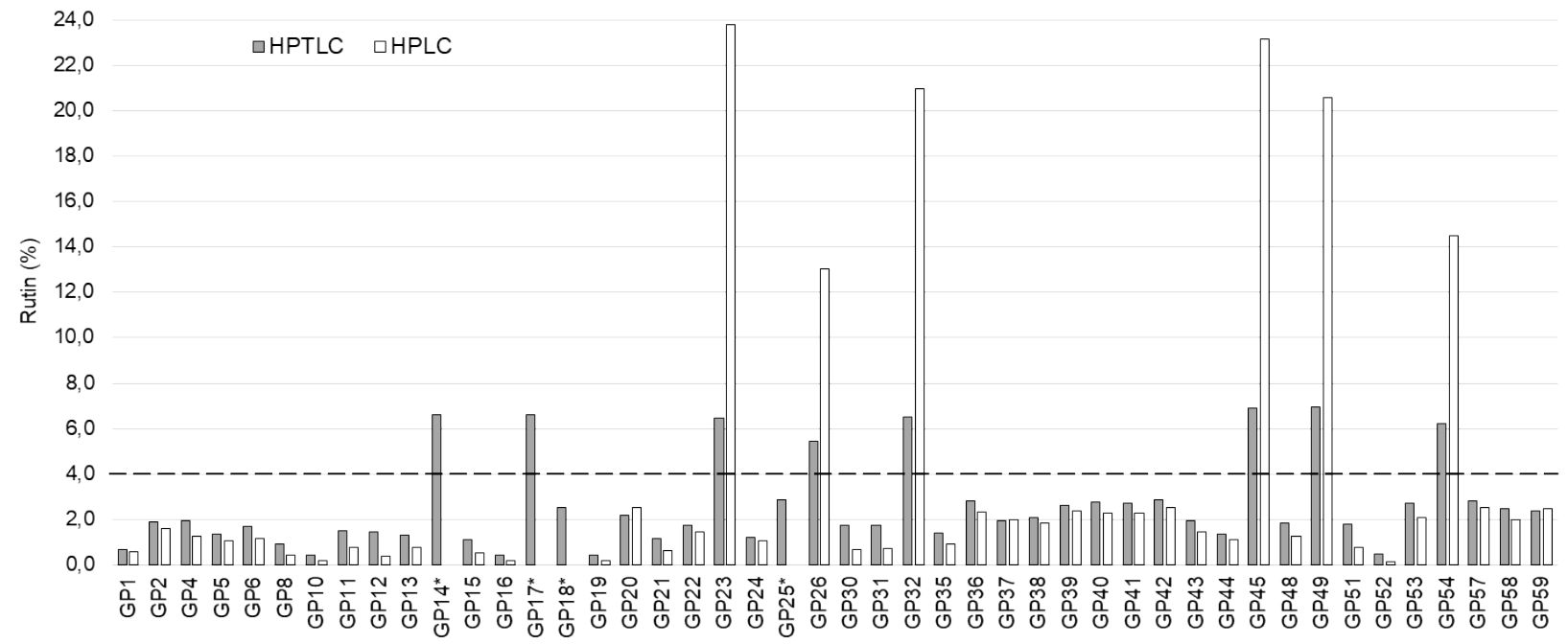

Fig. 4. Comparison of the results of the limit test for rutin in relation to the refined extracts contained in the products, obtained by the HPTLC ID method and by HPLC. Dashed line: maximum content of rutin (4\%) according to the USP powdered ginkgo extract monograph. * Not analysed by HPLC due to the limited amount of sample.

The HPTLC data were compared to those of HPLC. Results were in agreement regarding samples passing or failing the limit test. In six samples, the values from HPLC were considerably higher than those obtained by HPTLC. This was due to the limited dynamic range of the HPTLC detection resulting in saturation. Values below $4 \%$ were very similar with both methods. Thus, the HPTLC ID method can substitute the HPLC method in the limit test for rutin prescribed in the USP powdered ginkgo extract monograph.

\subsection{Limit test for quercetin}

The compliance to the limit test for quercetin was also investigated in the forty-six products using the HPTLC ID method. A ginkgo extract from HWI with known quercetin content was prepared to contain $0.5 \%$ of quercetin in the solution. This solution representing the samples in which 
quercetin is co-eluting with other zones (Fig. 1) was used instead of a standard solution of quercetin. After conversion of the image under UV $366 \mathrm{~nm}$ after derivatisation with NP into peak profiles, the height of the yellow/greenish peak at $R_{\mathrm{F}} 0.9$ (corresponding to $\mathrm{Q} / \mathrm{K} / \mathrm{I}$ and other zones) in the products was compared against that of the standard. Results are shown in Fig. 5.

Of the investigated products, only eighteen complied with the limit test for quercetin. Of those, seven contained mainly rutin (GP 14, 23, 30, 32, 45, 49 and 54) and one was adulterated with sophora fruit (GP26). Only ten had a fingerprint similar to GBE (GP 20, 36-43 and 59) (Fig. S1 and Fig. S2). This result was in agreement with the HPLC limit test for quercetin (Fig. 5). One product (GP54), which was found to be compliant after HPTLC analysis, was, however, found to contain more than $0.5 \%$ of quercetin when analysed by HPLC. Nevertheless, this sample was not compliant with the rutin limit test, and would thus fail in quality control.

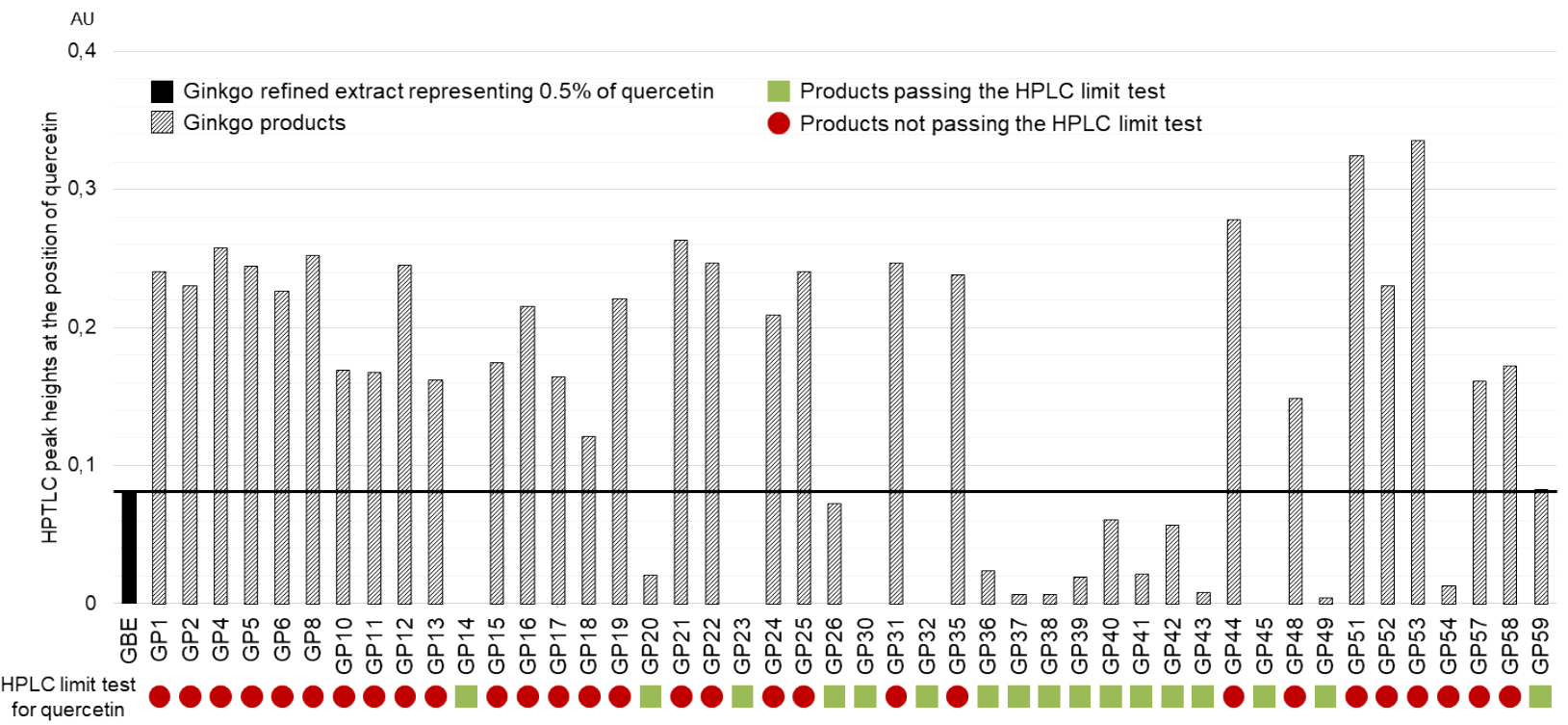

Fig. 5. Comparison of the quercetin zone intensity, expressed in AU, of forty-six ginkgo products containing refined extract with GBE representing $0.5 \%$ of quercetin.

An additional reversed phase HPTLC method (Table 1), which improves the separation of quercetin from the other two aglycones, was used for confirmation of results of the limit test for quercetin in all samples (Fig. S7). Peak profiles were generated from images under UV $366 \mathrm{~nm}$ obtained after derivatisation with NP and PEG reagents. The quercetin percentages were 
determined against a quercetin standard solution equivalent to $0.5 \%$ of the extract weight (Fig. 6 and Table S2). Results were in accordance to those obtained by HPLC.

The data using HPLC and HPLTC ID methods for the quercetin limit were comparable in $98 \%$ of the cases, and when combined with the other data, the HPTLC results lead to the same conclusion as those obtained by HPLC. Thus, the HPTLC ID method can be used instead of the USP HPLC method for determining the limit of quercetin. Samples with borderline results by HPTLC may be evaluated with other methodologies (HPLC or reversed phase HPTLC) for confirmation.

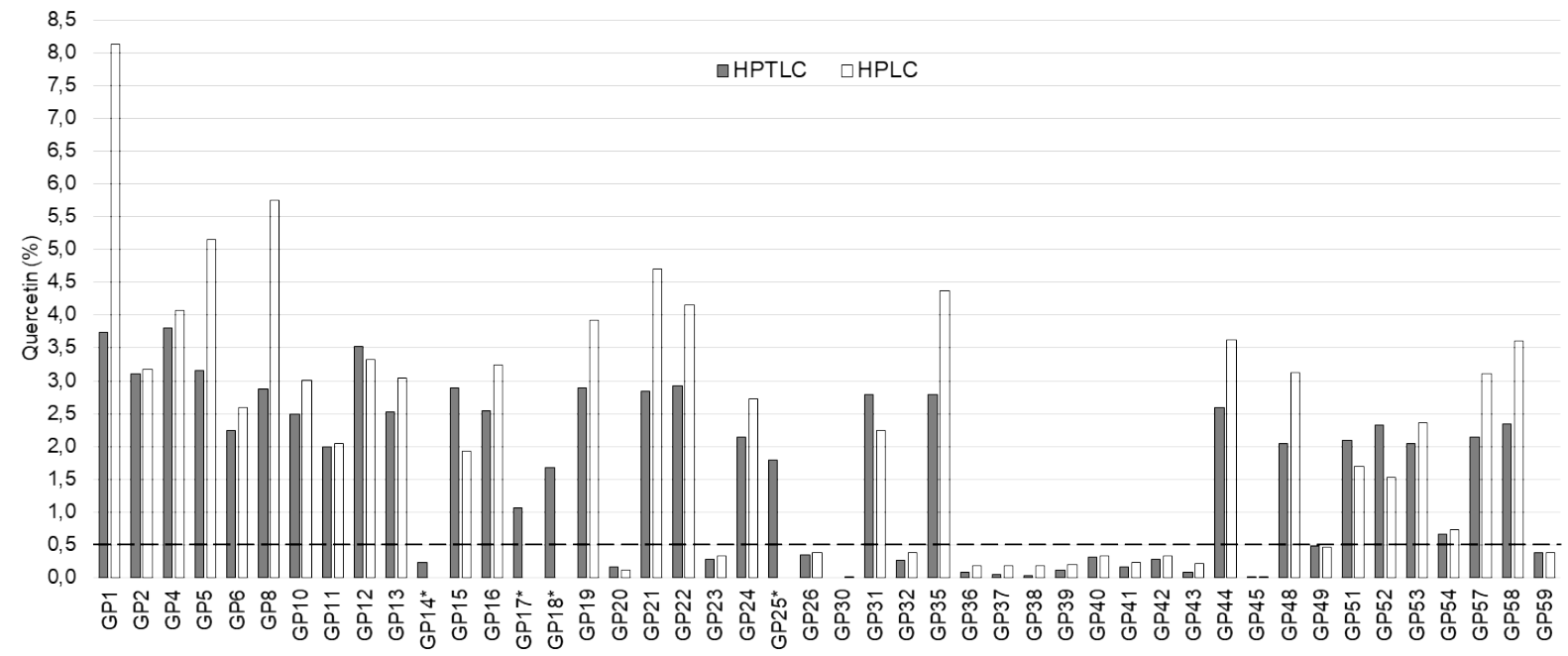

Fig. 6. Comparison of the results of the limit test for quercetin in relation to the refined extracts contained in the products, obtained by HPTLC reversed phase method and by HPLC. Dashed line: maximum content of quercetin $(0.5 \%)$ according to the USP powdered ginkgo extract monograph. * Not analysed by HPLC due to the limited amount of sample.

\subsection{HPTLC decision tree}

For better understanding of the significance of the proposed HPTLC method for the routine quality control of ginkgo products a decision tree has been designed (Fig. 7). It shows the sequence of interpretation of the fingerprints obtained with the different detections after a single HPTLC analysis. Thus, this decision tree can help the analyst to evaluate whether samples have the correct identity and whether they contain or not adulterants. 


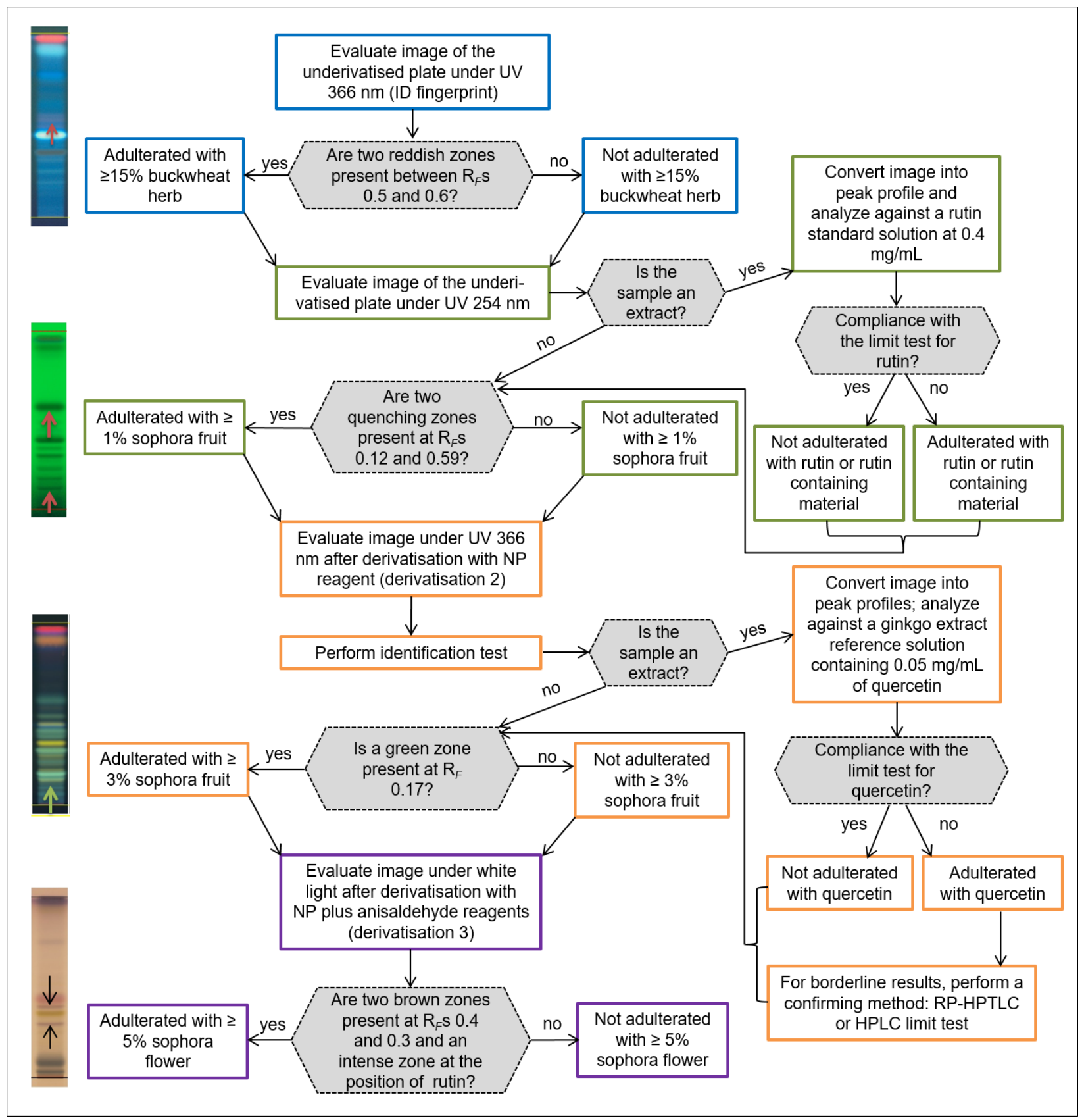

Fig. 7. Decision-tree about how to best perform comprehensive HPTLC fingerprinting for identification and detection of adulterations in ginkgo products.

\section{Conclusion}

This work provides a new pragmatic and cost-efficient approach to the quality control of ginkgo products, based on the "comprehensive HPTLC fingerprinting" concept. A single HPTLC analysis, carried out during identification, can also be used to detect adulteration with sophora fruit, sophora flower bud, buckwheat herb, quercetin, or rutin. Peak profiles generated from the images can be 
used to perform a limit test for quercetin and rutin without the need of an additional analysis if reference standards are applied at suitable levels. In this case HPTLC and HPLC data lead to the same conclusion. However, the HPTLC method cannot be considered valid for rutin or quercetin quantification purposes out of the limit test, which is only aimed to give a pass/fail decision.

Only eleven of the investigated fifty-nine GB products comply with specifications of the USP for identity, limits on rutin and quercetin, and content of total flavonoids. To the best of our knowledge, this is the largest study performed on commercial ginkgo products. Such a large percentage of poor quality and clearly adulterated products calls for quality control measures at earlier stages and throughout the value chains from the raw material to finished products (Booker and Heinrich 2016). For legitimate companies wanting to produce good quality products, HPTLC is able to provide important information concerning raw material acquisition, product development, the control of intermediate processing steps and final product compliance. However, much of the poor quality appears to be either deliberate (such as in the case of adulteration) or arising through an insufficient understanding of the manufacturing processes. In this context HPTLC can be a valuable tool used to highlight poor practice within the industry, provide evidence to regulatory bodies and ultimately to disseminate a greater awareness of product differences to the general public.

\section{Acknowledgments}

The authors wish to thank Mr. Enric Gibert for the technical support with the HPLC analyses, Mr. Christian Goy for providing part of the samples, Mr. Sidney Sudberg (Alkemist Lab) for providing references samples, Dr. Maged Sharaf for proof reading the article. Some of the initial samples were purchased with funds provided by the BBC, UK as a part of their programme 'Trust Me - I am a Doctor'. The authors are also grateful to the help of Fundación Maria Francisca de Roviralta for the acquisition of laboratory equipment for the University of Barcelona.

\section{Author contributions}

DAF did most of the experimental work with the collaboration of ER, SC and RV. MH and AB provided a big part of the samples. ER and SC supervised the work. DAF, ER and SC were responsible for writing the manuscript. All authors proof read manuscript and made contributions to the final version. 


\section{Conflict of interest}

The authors declare no competing financial interests.

\section{Supporting information}

Figures $\mathrm{S} 1$ to $\mathrm{S} 7$ and Tables $\mathrm{S} 1$ to $\mathrm{S} 3$ are provided as supporting information.

\section{References}

Avula, B., Sagi, S., Gafner, S., Upton, R., Wang, Y.H., Wang, M., Khan, I.A., 2015. Identification of Ginkgo biloba supplements adulteration using high performance thin layer chromatography and ultra high performance liquid chromatography-diode array detectorquadrupole time of flight-mass spectrometry. Anal. Bioanal. Chem., 407(25), 7733-7746. https://doi.org/10.1007/s00216-015-8938-1

Booker, A., Frommenwiler, D., Reich, E., Horsfield, S., Heinrich, M., 2016. Adulteration and poor quality of Ginkgo biloba supplements. J. Herbal Med., 6(2). https://doi.org/10.1016/j.hermed.2016.04.003

Booker, A., Heinrich, M., 2016. Value chains of botanicals and herbal medicinal products: a European perspective. HerbalGram, 112, 40-45.

Chandra, A., Li, Y., Rana, J., Persons, K., Hyun, C., Shen, S., Mulder, T., 2011. Qualitative categorization of supplement grade Ginkgo biloba leaf extracts for authenticity. J. Funct. Foods, 3(2), 107-114. https://doi.org/10.1016/j.jff.2011.03.004

Czigle, S., Tóth, J., Jedlinszki, N., Háznagy-Radnai, E., Csupor, D., Tekel’ová, D., 2018. Ginkgo biloba Food Supplements on the European Market - Adulteration Patterns Revealed by Quality Control of Selected Samples. Planta Med., 84(6-07), 475-482. https://doi.org/10.1055/a-0581-5203

Drieu, K, Jaggy, H., 2000. History, development and constituents of EGb 761, in van Beek, T.A. (Ed.), Ginkgo biloba, Chapter 15. Harwood Academic Publishers, Amsterdam, pp. 267-277.

EDQM (European Directorate for the Quality of Medicines and Health Care), 2018. Monograph on Ginkgo dry extract, refined and quantified. European Pharmacopoeia. $9^{\text {th }}$ Ed. Council of Europe, Strasbourg. 
European Medicines Agency, 2015. European Union herbal monograph on Ginkgo biloba L., folium. Document EMA/HMPC/321097/2012. European Medicines Agency, London. https://www.ema.europa.eu/documents/herbal-monograph/final-european-union-herbalmonograph-ginkgo-biloba-1-folium_en.pdf

Franz, C., Chizzola, R., Novak, J., Sponza, S. (2011). Botanical species being used for manufacturing plant food supplements (PFS) and related products in the EU member states and selected third countries. Food Funct., 2(12), 720-730. https://doi.org/10.1039/c1fo10130g

Frommenwiler, D. A., Kim, J., Yook, C.-S., Tran, T. T. T., Cañigueral, S., Reich, E., 2018. Comprehensive HPTLC Fingerprinting for Quality Control of an Herbal Drug - The Case of Angelica gigas Root. Planta Med., 84(6-07), 465-474. https://doi.org/10.1055/a-0575-4425

Gafner, S., 2018. Adulteration of Ginkgo biloba Leaf Extract. Botanical Adulterants Bulletin, January, 1-8.

He, K., Roller, M., 2011. The Use of Nuclear Magnetic Resonance Spectroscopy for the Identification of Biomarkers for Quality Control of Plant Extracts, in Dayan, N., Kromidas, L. (Eds.), Formulating, Packaging, and Marketing of Natural Cosmetic Products. Wiley, Hoboken, NJ, pp. 361-385.

Heinrich, M., 2010. Ethnopharmacology and Drug Discovery, in: Mander, L., Lui, H.-W., (Eds.), Comprehensive Natural Products II Chemistry and Biology, Chapter 3.12. Elsevier, Oxford, pp. 351-381.

López-Gutiérrez, N., Romero-González, R., Vidal, J.L. M., Frenich, A.G., 2016. Quality control evaluation of nutraceutical products from Ginkgo biloba using liquid chromatography coupled to high resolution mass spectrometry. J. Pharm. Biomed. Anal., 121, 151-160. https://doi.org/10.1016/j.jpba.2016.01.021

Ma, Y. C., Mani, A., Cai, Y., Thomson, J., Ma, J., Peudru, F., Chen, S., Luo, M., Zhang, J., Chapman, R.G., Shi, Z.T., 2016. An effective identification and quantification method for Ginkgo biloba flavonol glycosides with targeted evaluation of adulterated products. Phytomedicine, 23(4), 377-387. https://doi.org/10.1016/j.phymed.2016.02.003

Tawab, M., Krzywon, M., Schubert-Zsilavecz, M., 2010. Nahrungsergänzungsmittel mit Ginkgo unter der Lupe. Pharm. Ztg. 155(20),1994-1999 
The United States Pharmacopoeia (USP), 2016a. Monograph for ginkgo, in United States Pharmacopoeia and National Formulary USP38-NF33. United States Pharmacopoeial Convention, Rockville, MD, USA, pp. 6668-6671.

The United States Pharmacopoeia (USP), 2016b. Monograph for powdered ginkgo extract, in United States Pharmacopoeia and National Formulary USP39-NF34. United States Pharmacopoeial Convention, Rockville, MD, USA, pp.6671-6674.

The United States Pharmacopoeia (USP), 2017. Chapter 203: High-Performance Thin-Layer Chromatography procedure for identification of articles of botanical origin, in United States Pharmacopoeia and National Formulary USP40-NF35. United States Pharmacopoeial Convention, Rockville, MD, USA, pp. 258-260.

van Beek, T.A., Montoro, P., 2009. Chemical analysis and quality control of Ginkgo biloba leaves, extracts, and phytopharmaceuticals. J. Chromatogr. A, 1216(11), 2002-2032. https://doi.org/10.1016/j.chroma.2009.01.013

Wohlmuth, H., Savage, K., Dowell, A., Mouatt, P., 2014. Adulteration of Ginkgo biloba products and a simple method to improve its detection. Phytomedicine, 21(6), 912-918. https://doi.org/10.1016/j.phymed.2014.01.010

Yang, J., Wang, A. Q., Li, X.J., Fan, X., Yin, S.S., Lan, K., 2016. A chemical profiling strategy for semi-quantitative analysis of flavonoids in Ginkgo extracts. J. Pharm. Biomed. Anal., 123, 147-154. https://doi.org/10.1016/j.jpba.2016.02.017 